\title{
Quadratic Optical Frequency Combs
}

\author{
Stefan Wabnitz \\ Dept. of Information, Electronics and Telecom Engineering \\ Sapienza University of Rome \\ Rome, Italy \\ stefan.wabnitz@uniroma1.it
}

\author{
Miro Erkintalo \\ Department of Physics \\ University of Auckland \\ Auckland, New Zealand \\ m.erkintalo@auckland.ac.nz
}

\author{
Tobias Hansson \\ Department of Physics, Chemistry and Biology \\ Linköping University \\ Linköping, Sweden \\ tobias.hansson@liu.se
}

\author{
Simona Mosca, Maria Parisi, \\ Iolanda Ricciardi, and Maurizio De Rosa \\ CNR-INO \\ Istituto Nazionale di Ottica \\ Pozzuoli, Italy \\ maurizio.derosa@ino.it
}

\begin{abstract}
Optical frequency comb sources based on quadratic nonlinearities provide an interesting alternative to Kerr combs in terms of reduced pump power requirements and extended spectral coverage. We review theory and recent experiments of quadratic optical frequency combs based on second-harmonic generation and optical parametric oscillation.

Index Terms-Optical frequency combs, second-harmonic generation, optical parametric oscillation, optical solitons
\end{abstract}

\section{INTRODUCTION}

High-precision optical frequency combs (OFCs), often referred to as optical rulers, had a strong impact on metrology and spectroscopy in the last decade. Recent advances in the technology of optical micro-cavities contribute to the development of chip-scale OFC sources [1]. The mechanism for comb formation in microcombs is the cubic or Kerr nonlinearity of the waveguide material, which leads to dissipative modulation instability [2], followed by cascaded four-wave mixing (FWM). In spite of the many exciting achievements in the field of micro-combs, namely optical frequency combs based on high-Q micro-resonators, several open questions and challenges remain. For example, the relatively high continuouswave $(\mathrm{CW})$ pump power, the poor conversion efficiency from the $\mathrm{CW}$ pump to the comb, the complex stabilization required for coherent soliton combs, and the scarcity of available pumps in some spectral regions such as the visible and the midinfrared. Some of these issues can be solved by exploiting OFC generation mechanisms based on quadratic, as opposed to cubic, nonlinear resonators. We overview here recent progress in the research of quadratic OFCs.

\section{SHG COMBS}

Quadratic nonlinear processes can be exploited for the frequency conversion of near-infrared OFCs into the visible or mid-infrared.

\section{A. Singly-resonant case}

In 2015, Ricciardi et al. experimentally demonstrated the direct generation of OFCs in a cavity-enhanced second- harmonic-generation (SHG) system [3]. Two OFCs were simultaneously generated around the fundamental (FF) pump frequency at $1064 \mathrm{~nm}$, with a spectral bandwidth up to about $10 \mathrm{~nm}$, and its second harmonic (SH). Different regimes of generation were observed, depending on the SHG phasematching condition. A simple coupled three-wave model was developed to describe the observations, revealing the analogy with FWM-based OFC generation in cubic resonators. This model was later generalized to describe a many-mode comb, and a stability analysis of the different cavity field regimes was presented [4].

In 2016, Leo et al. derived a single time-domain, mean-field equation that fully models the temporal and spectral dynamics of OFC generation in singly resonant, cavity-enhanced SHG systems [5]. This study permitted to show that the temporal walk-off between the FF and the SH plays a decisive role in activating the comb. A linear stability analysis coupled with numerical simulations revealed a new type of modulation instability (MI), leading to OFCs and their associated timedomain dissipative structures, in excellent agreement with the experiments [3].

By using the temporal mean-field equation for the FF with a noninstantaneous nonlinear response function, Hansson et al. could explore the rich temporal and spectral dynamics of quadratic comb generation, continuous wave bistability, and modulational instabilities [6]. Temporal walk-off between the FF and the SH leads to MI even in the absence of groupvelocity dispersion. The time-domain model was compared with the modal expansion approach [4], showing that the two models provide very similar predictions and become exactly equivalent in the limit that absorption losses and groupvelocity dispersion at the FF are neglected.

\section{B. Doubly-resonant case}

OFC generation in doubly-resonant intracavity SHG was also theoretically analyzed by Leo et al. [7]. Despite the large temporal walk-off characterizing realistic cavity systems, the comb generation dynamics can be accurately modeled by using 
a pair of coupled mean-field equations. A stability analysis of steady-state cavity $\mathrm{CW}$ solutions revealed that walk-off may enable to onset of a new type of MIs. Numerical simulations predict emergence of temporal patterns corresponding to coherent optical frequency combs. Under typical conditions, the two coupled equations may be reduced to a single mean-field equation, similar to that describing the dynamics of the singlyresonant-cavity SHG, so that a simple analytical expression for the MI gain can be obtained [5].

A subsequent theoretical study by Hansson et al. permitted to identify different dynamical regimes, and predicted that, in the absence of temporal walk-off between the FF and the $\mathrm{SH}$, doubly-resonant intracavity SHG supports the propagation of both bright and dark time-localized cavity solitons [8]. These soliton solutions are found to have analogous properties to Kerr cavity solitons, in particular they correspond to stable coherent OFCs, which suggests that quadratic frequency combs may be a viable alternative to Kerr combs.

\section{OPO COMBS}

In 2018, Mosca et al. reported on the formation of quadratic combs inside an optical parametric oscillator (OPO) [9]. Namely it was shown that OFCs were generated around half of the pump frequency in a singly-resonant, CW-driven OPO. The OPO dynamics could also be modeled by means of a single time-domain mean-field equation, identifying previously unknown dynamical regimes, induced by MIs, which lead to OFC formation. The analysis of the coherence properties of the simulated spectra showed the existence of correlated and phase-locked combs.

Parra-Rivas et al. have recently theoretically investigated the formation of localized temporal dissipative structures, and their corresponding OFCs, in doubly-resonant dispersive OPOs [10]. A nonlocal mean field model was derived, predicting domain-wall locking as a mechanism for the formation of stable coherent OFCs.

\section{Multi-OCTAVE COMBS}

In situations where multiple quadratic processes occur, it may be necessary to numerically study OFC generation by means of a single envelope equation. Hansson et al. investigated the generation of OFCs ranging from the visible to the mid-infrared spectral regions in a radially poled lithium niobate whispering gallery resonator with both quadratic and cubic nonlinearities [11]. It was found that MIs may lead to the formation of coupled comb arrays extending over multiple octaves.

\section{AlGAAs COMBS}

OFC generation in cavity-enhanced SHG can be achieved by directional quasi-phase-matching in AlGaAs waveguide resonators. Parisi et al. have shown that internally pumped OPO may trigger comb formation [12]. The study showed that efficient generation of coherent OFCs is possible with threshold powers in the microwatt range, and path lengths up to several millimeters, thus enabling the practical realization of new on-chip frequency comb synthesizers, fully integrable in complex photonic circuits.

\section{ACKNOWLEDGMENT}

We acknowledge funding from: Ministero dell'Istruzione, Università e Ricerca (MIUR) (2015KEZNYM-NEMO); H2020 European Research Council (ERC) (757800).

\section{REFERENCES}

[1] A. Pasquazi, M. Peccianti, L. Razzari, D. J. Moss, S. Coen, M. Erkintalo, Y. K. Chembo, T. Hansson, S. Wabnitz, P. Del'Haye, X. Xue, A. M. Weiner, and R. Morandotti, "Micro-combs: A novel generation of optical sources," Physics Reports, vol. 729, pp. 1 - 81, 2018. [Online]. Available: http://www.sciencedirect.com/science/article/pii/S0370157317303253

[2] M. Haelterman, S. Trillo, and S. Wabnitz, "Dissipative modulation instability in a nonlinear dispersive ring cavity," Optics Communications, vol. 91, no. 5-6, pp. 401-407, 1992.

[3] I. Ricciardi, S. Mosca, M. Parisi, P. Maddaloni, L. Santamaria, P. De Natale, and M. De Rosa, "Frequency comb generation in quadratic nonlinear media," Phys. Rev. A, vol. 91, p. 063839, Jun 2015. [Online]. Available: https://link.aps.org/doi/10.1103/PhysRevA.91.063839

[4] S. Mosca, I. Ricciardi, M. Parisi, P. Maddaloni, L. Santamaria, P. D. Natale, and M. D. Rosa, "Direct generation of optical frequency combs in $\chi^{2}$ nonlinear cavities," Nanophotonics., vol. 5, no. 2, pp. 361-331, Jun 2016.

[5] F. Leo, T. Hansson, I. Ricciardi, M. De Rosa, S. Coen, S. Wabnitz, and M. Erkintalo, "Walk-off-induced modulation instability, temporal pattern formation, and frequency comb generation in cavity-enhanced second-harmonic generation," Phys. Rev. Lett., vol. 116, p. 033901, Jan 2016. [Online]. Available: https://link.aps.org/doi/10.1103/PhysRevLett.116.033901

[6] T. Hansson, F. Leo, M. Erkintalo, S. Coen, I. Ricciardi, M. De Rosa, and S. Wabnitz, "Singly resonant second-harmonic-generation frequency combs," Phys. Rev. A, vol. 95, p. 013805, Jan 2017. [Online]. Available: https://link.aps.org/doi/10.1103/PhysRevA.95.013805

[7] F. Leo, T. Hansson, I. Ricciardi, M. De Rosa, S. Coen, S. Wabnitz, and M. Erkintalo, "Frequency-comb formation in doubly resonant second-harmonic generation," Phys. Rev. A, vol. 93, p. 043831, Apr 2016. [Online]. Available: https://link.aps.org/doi/10.1103/PhysRevA.93.043831

[8] T. Hansson, P. Parra-Rivas, M. Bernard, F. Leo, L. Gelens, and S. Wabnitz, "Quadratic soliton combs in doubly resonant secondharmonic generation," Opt. Lett., vol. 43, no. 24, pp. 6033-6036, Dec 2018. [Online]. Available: http://ol.osa.org/abstract.cfm?URI=ol-43-246033

[9] S. Mosca, M. Parisi, I. Ricciardi, F. Leo, T. Hansson, M. Erkintalo, P. Maddaloni, P. De Natale, S. Wabnitz, and M. De Rosa, "Modulation instability induced frequency comb generation in a continuously pumped optical parametric oscillator," Phys. Rev. Lett., vol. 121, p. 093903, Aug 2018. [Online]. Available: https://link.aps.org/doi/10.1103/PhysRevLett.121.093903

[10] P. Parra-Rivas, L. Gelens, T. Hansson, S. Wabnitz, and F. Leo, "Frequency comb generation through the locking of domain walls in doubly resonant dispersive optical parametric oscillators," Opt. Lett., vol. 44, no. 8, pp. 2004-2007, Apr 2019. [Online]. Available: http://ol.osa.org/abstract.cfm?URI=ol-44-8-2004

[11] T. Hansson, F. Leo, M. Erkintalo, J. Anthony, S. Coen, I. Ricciardi, M. D. Rosa, and S. Wabnitz, "Single envelope equation modeling of multi-octave comb arrays in microresonators with quadratic and cubic nonlinearities," J. Opt. Soc. Am. B, vol. 33, no. 6, pp. 1207-1215, Jun 2016. [Online]. Available: http://josab.osa.org/abstract.cfm?URI=josab33-6-1207

[12] M. Parisi, N. Morais, I. Ricciardi, S. Mosca, T. Hansson, S. Wabnitz, G. Leo, and M. D. Rosa, "AlGaAs waveguide microresonators for efficient generation of quadratic frequency combs," J. Opt. Soc. Am. $B$, vol. 34, no. 9, pp. 1842-1847, Sep 2017. [Online]. Available: http://josab.osa.org/abstract.cfm?URI=josab-34-9-1842 